\title{
Active Matter Commensuration and Frustration Effects on Periodic Substrates
}

\author{
C. Reichhardt and C. J. O. Reichhardt \\ Theoretical Division and Center for Nonlinear Studies, \\ Los Alamos National Laboratory, \\ Los Alamos, New Mexico 87545, USA
}

(Dated: November 5, 2020)

\begin{abstract}
We show that self-driven particles coupled to a periodic obstacle array exhibit novel active matter commensuration effects that are absent in the Brownian limit. As the obstacle size is varied for sufficiently large activity, a series of commensuration effects appear in which the motility induced phase separation produces commensurate crystalline states, while for other obstacle sizes we find frustrated or amorphous states. The commensuration effects are associated with peaks in the amount of six-fold ordering and the maximum cluster size. When a drift force is added to the system, the mobility contains peaks and dips similar to those found in transport studies for commensuration effects in superconducting vortices and colloidal particles.
\end{abstract}

Commensuration effects arise in a variety of hard and soft matter systems when an assembly of particles is coupled to a periodic substrate with a spacing that matches the average interparticle spacing. Such effects occur for the ordering of atoms or molecules on surfaces [1 3] , vortices in superconductors or Bose-Einstein condensates with periodic pinning arrays [4 7], colloidal particles on optical trap arrays [8 -10] or patterned surfaces [11], and cold atoms on optical lattices [12]. Conversely, if the particle assembly cannot fit within the constraints imposed by the substrate, then frustration can cause the disordering of the system or the formation of localized defects such as kinks or anti-kinks [13, 14]. Commensuration effects also strongly modify the transport properties under an applied drive in these systems, producing reduced transport or enhanced pinning when a commensuration occurs and generating a series of peaks or dips in the transport coefficients as the parameters are varied [5, 6, 13 -17].

Coupling of active matter or self-driven particles to a substrate 18, 19] has been realized in numerous experiments 18 22]. Many active particles have only short range repulsive interactions, so the system forms a uniform liquid at lower densities in the non-active or Brownian limit; however, when activity is present, the particles undergo a self-clustering or motility induced phase separation into a high density crystalline phase surrounded by a low density gas [20, 21, 23 25]. Although there have been various methods proposed for coupling an active matter system to random [19, 22, 26 32] or periodic obstacle arrays 33 38], the possible commensuration effects that could occur on a periodic substrate in active systems have not been considered before now. For a twodimensional (2D) system of disks in the Brownian or zero activity regime, commensuration effects do not arise until the disk density $\phi$ is high enough for all of the disks to touch each other, so for a nonactive system at $\phi<0.8$, commensuration effects should be absent. Additionally, since thermal effects typically wash out commensuration effects 15, it might be expected that active matter systems would not exhibit commensuration effects.

Here we examine a 2D active matter system of selfpropelled run-and-tumble disks interacting with a square array of obstacles. For certain obstacle sizes, we find that the system can undergo a strong motility-induced phase transition into a crystalline state that is commensurate with the obstacle lattice and that coexists with a low density gas. For other obstacle sizes, the motility-induced phase separation produces an amorphous crystal due to a frustration effect caused by a mismatch between the active disk spacing and the obstacle spacing. The spacing of the disks in the motility-induced dense phase is key in determining whether commensurate or incommensurate behavior occurs. The commensuration effects produce peaks in the size of the largest cluster and in the amount of sixfold ordering. A variety of different commensurate states appear, including states with local square ordering, aligned states, and sliding crystalline states. Under an applied drift force, the transport is a strongly nonmonotonic function of the obstacle size and exhibits dips at commensurate states as well as peaks at incommensurate or frustrated states. These commensuration effects are absent in the Brownian limit and become stronger for increasing activity or longer run times.

Simulation and System - We model a 2D system of active run-and-tumble disks of density $\phi_{a}$ interacting with a periodic array of obstacles composed of posts of diameter $d$ and lattice constant $a$. The overdamped equation of motion for an active disk $i$ is given by

$$
\alpha_{d} \mathbf{v}_{i}=\mathbf{F}_{i}^{d d}+\mathbf{F}_{i}^{m}+\mathbf{F}_{i}^{o b s}+\mathbf{F}_{i}^{D}
$$

where the damping constant $\alpha_{d}=1.0$, and $\mathbf{r}_{i}$ and $\mathbf{v}_{i}=d \mathbf{r}_{i} / d t$ are the position and velocity of disk $i$. For the disk-disk interaction force $\mathbf{F}_{i}^{d d}$, we use a harmonic repulsion with spring constant $k_{a}$ and disk radius $r_{a}$, so that the disk diameter is $d_{a}=2 r_{a}$. We set $k_{a}=150$, which is large enough to keep the disk-disk overlap in our study below one percent. The disk-obstacle force 


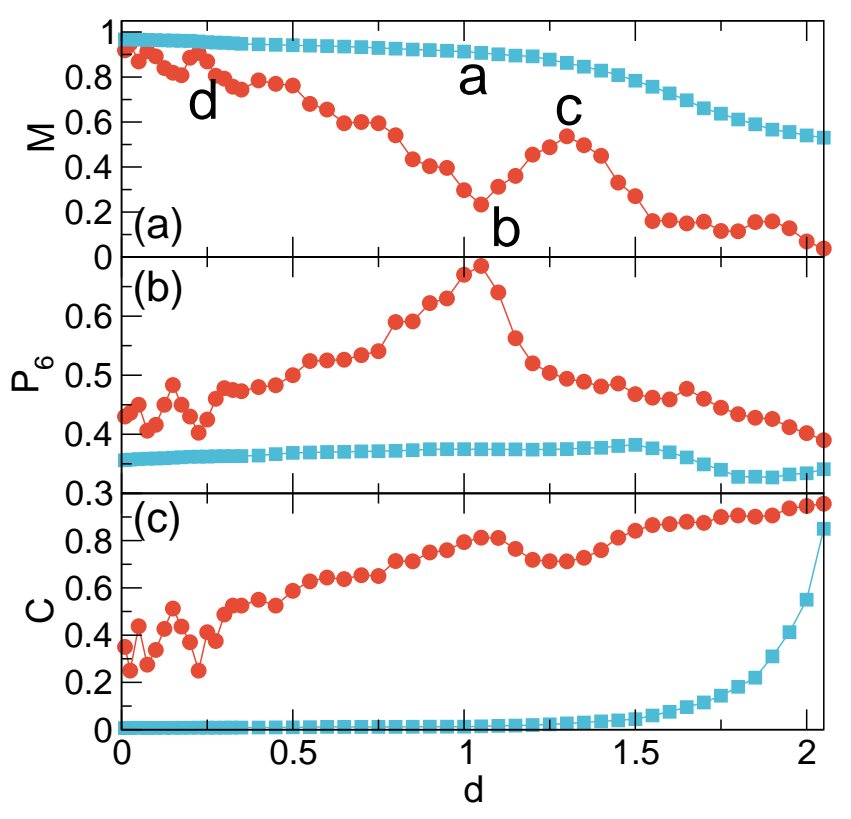

FIG. 1. Behavior of an active disk system with $d_{a}=0.9$ for a run length of $l_{r}=0.025$ (squares) in the Brownian limit and $l_{r}=175$ (circles) in the active limit. (a) Mobility $M$ vs obstacle diameter $d$. (b) Fraction of six-fold coordinated particles $P_{6}$ vs $d$. (c) Size of the largest cluster $C$ vs $d$. The letters a-d in panel (a) indicate the points corresponding to the images in Fig. 2 ,

$\mathbf{F}^{\text {obs }}$ is also modeled as a harmonic potential. The active disk coverage is $\phi_{a}$ and the combined coverage of the disks and obstacles is $\phi_{\text {tot }}$. For the self-propulsion of the active disks $\mathbf{F}^{m}$, a force $F_{M}$ is applied in a randomly chosen direction for a run time of $\tau_{l}$, after which the motor force instantaneously reorients to a new randomly chosen direction for the next run time. We characterize the system by the run length $r_{l}=F_{m} \tau_{l}$, the distance an isolated active particle would move during the run time $\tau_{l}$. We also consider the effects of an external drive $\mathbf{F}^{D}=F_{D} \hat{\mathbf{x}}$ and measure the mobility $M$ using the average velocity in the driving direction, $\left\langle V_{x}\right\rangle=\sum_{i=1}^{N^{d}} \mathbf{v} \cdot \mathbf{x}$. We define $M=\left\langle V_{x}\right\rangle / V_{\text {free }}$ where $V_{\text {free }}$ is the average velocity that would appear under the same driving force in the absence of any obstacles. We fix $a=3.0$ and $F_{D}=0.2$, and vary $d_{a}, d$, and $r_{l}$.

Results- In Fig. 1 $(\mathrm{a}, \mathrm{b}, \mathrm{c})$ we plot the mobility $M$, the fraction of six-fold coordinated particles $P_{6}$, and the fraction of particles in the largest cluster $C$ versus the obstacle diameter $d$ for a system with $\phi_{a}=0.32$ and $d_{a}=0.9$. We show two run length values: $l_{r}=0.025$, where the system is in the Brownian limit, and $l_{r}=175$, the active limit where an obstacle free system would exhibit motility induced phase separation. For the short run length of $l_{r}=0.025, M$ has an initial value near 1.0 and exhibits a monotonic decrease with increasing $d$, while $P_{6}$ is mostly flat and $C$ starts to increase once $d>1.5$. For the active
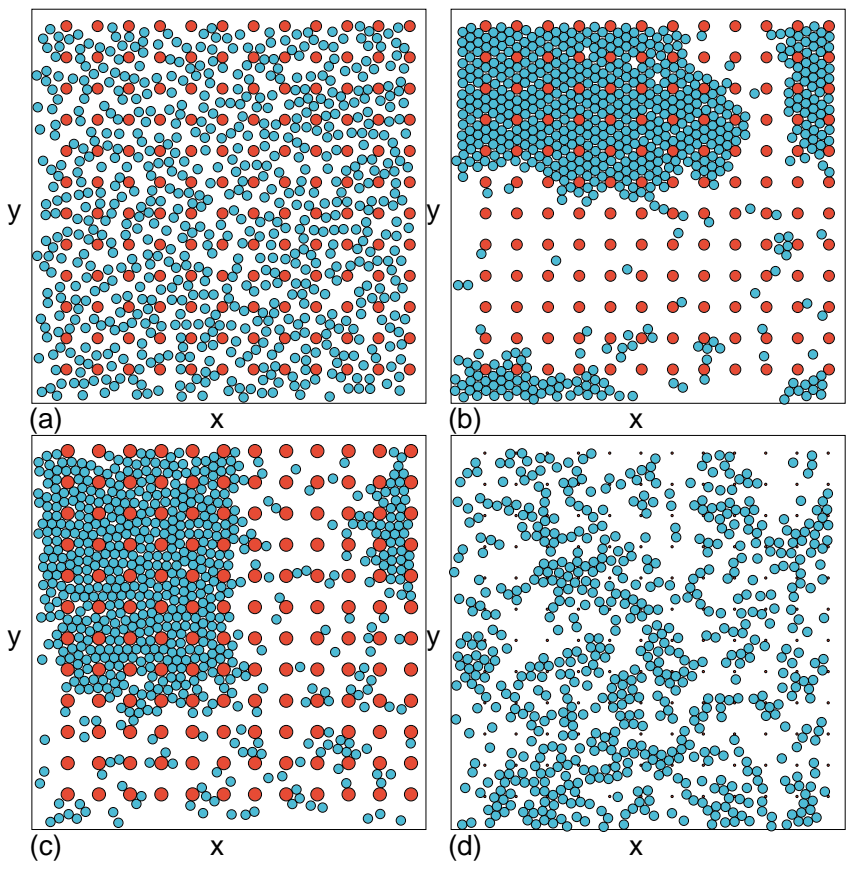

FIG. 2. Snapshots of the active disk positions (blue circles) and the obstacles (red circles) for the system in Fig. 1 with $d_{a}=0.9$. (a) A uniform liquid at $d=1.05$ and $l_{r}=0.025$. (b) At $d=1.05$ and $l_{r}=175$, there is a phase separated state in which the dense regions form a commensurate solid. (c) At $d=1.3$ and $l_{r}=175$, there is a phase separated amorphous or frustrated state. (d) A frustrated state at $d=0.225$ and $l_{r}=175$.

limit of $l_{r}=175, M$ also has an initial value near 1.0 but changes nonmonotonically with increasing $d$, showing a pronounced dip near $d=1.05$ which correlates with a peak in $P_{6}$ and a smaller peak in $C$. There is also a peak in $M$ near $d=1.3$ that is associated with a drop in $P_{6}$ and a smaller dip in $C$. Additional features include a peak in $M$ near $d=0.2$ and a smaller peak near $d=1.75$.

In Fig. 2(a) we show a snapshot of the active particles and the obstacles for the system in Fig. 1 at $l_{r}=0.025$ and $d=1.05$, where a uniform liquid state appears. By comparison, in Fig. 2(b) a sample with $l_{r}=175$ at $d=1.05$ forms a phase separated state of high density coexisting with a low density gas. This combination of parameters corresponds to the peak in $P_{6}$ and the dip in $M$ in Fig. 1. In Fig. 3(a) we show a blowup of the high density region from Fig. 2(b), indicating more clearly that the system forms a triangular lattice which is commensurate with the underlying square array. Figure 2(c) illustrates the system in Fig. 1 at $l=175$ and $d=1.3$, corresponding to a local minimum in $M$ and a drop in $P_{6}$. Although the system still shows clustering, the structure of the active disks in the dense region is now amorphous, as shown more clearly in Fig. 3(b). The disorder is produced by a frustration effect that arises when the natural spacing of the active crystal does not 

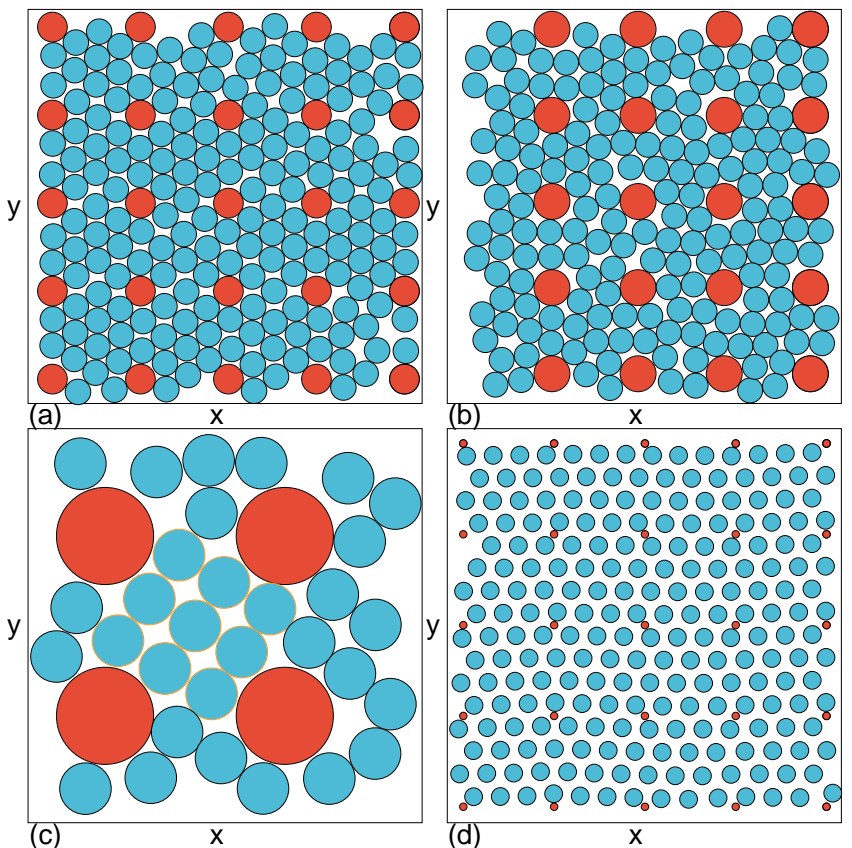

(b)

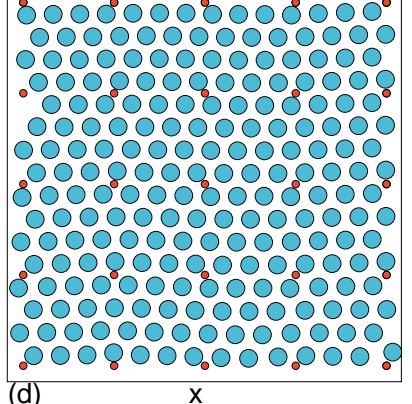

(d)

FIG. 3. Snapshots of the active disk positions (blue circles) and the obstacles (red circles) for the system in Fig. 10 with $d_{a}=0.9$. (a) A blow up of the dense region in Fig. 2 (b) at $d=1.05$ and $l_{r}=175$, showing triangular commensurate ordering. (b) A blow up of the dense region in Fig. 2(c) at $d=1.3$ and $l_{r}=175$, which is in an amorphous state. (c) A blow up illustrating the local square ordering at $d=1.7$ and $l_{r}=175$. (d) The full system at $d=0.15$ and $l_{r}=175$ showing a sliding crystal phase. For clarity, the size of the mobile disks has been reduced in panel (d).

match the spacing of the interstitial region between the obstacles. The dip in $M$ is similar to the drop in motion or decrease in the critical depinning force found in nonactive commensurate systems at incommensurate densities [5, 6, 13 15]. In the non-active systems, the commensurate crystalline states have a higher shear modulus and can be more strongly pinned by the obstacles. In contrast, for the frustrated system the shear modulus is reduced, permitting the particles to move more easily and producing minima in the depinning force of the incommensurate state. Near $d=0.2$ for the active system in Fig. 1] a peak in $M$ and a dip in $P_{6}$ appear at another incommensurate region where the disks are disordered, as shown in Fig. 2(d) for $d=0.225$.

For $d>1.5$, a distinctive type of active cluster appears which has local square short-range ordering within a single plaquette. These clusters are associated with a drop in $M$ and an increase in $C$. An example of the $d=1.7$ clustered state appears in Fig. 3(c), where a single plaquette with local square ordering is highlighted. As $d$ increases further, other types of commensurate crystals can occur.

In the active system near $d=0.15$, where there is a
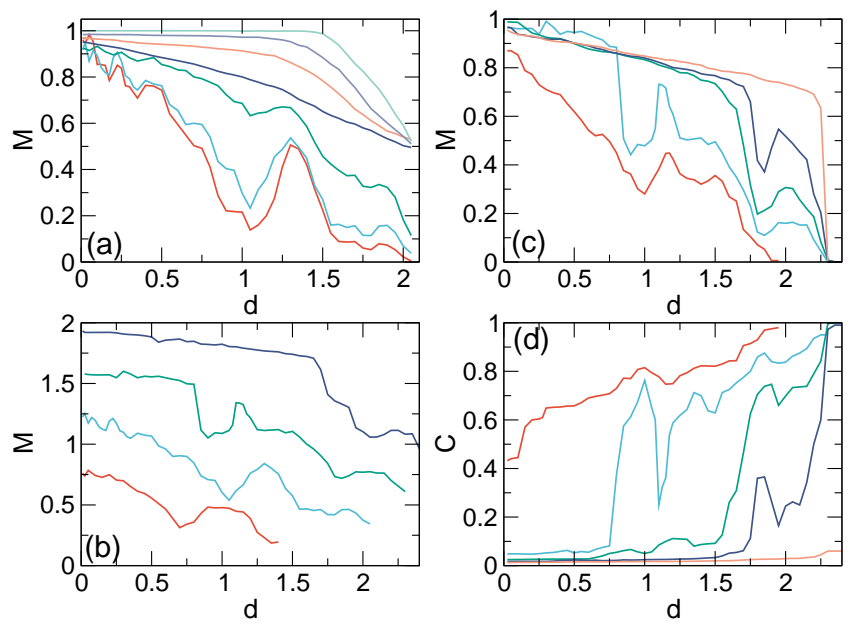

FIG. 4. (a) $M$ vs $d$ for the system in Fig. 1 for $l_{r}=0.00025$, $0.1,0.025,0.25,40,175$, and 1750 , from top to bottom. (b) $M$ vs $d$ for $l_{r}=175$ at $d_{a}=0.7,0.8,0.9$, and 1.0, from top to bottom, corresponding to $\phi_{a}=0.195,0.254,0.32$, and 0.4 . For clarity, the first three curves are shifted up by $0.915,0.61$, and 0.305 , respectively, on the $M$ axis. (c) $M$ vs $d$ for samples with $d_{a}=0.8$ and $l_{r}=175$ at varied disk density $\phi_{a}=$ $0.00212,0.09,0.17,0.254$, and 0.332 , from top to bottom. (d) The corresponding fraction of particles in the largest cluster $C$ vs $d$ for the system in panel (c).

smaller peak in $C$, the disks form a sliding crystalline state where the commensuration effect is determined by the number of rows of active disks that can fit between adjacent rows of obstacles, as shown in Fig. 3(d) for four rows of disks. The sliding crystal exhibits intermittent jumping between crystal and disordered states, causing the value of $P_{6}$ to be reduced compared to the commensurate state which appears at higher $d$. If we consider a random array of obstacles, we do not observe any commensurate effects but instead find a monotonic decrease of the mobility with increasing $d$.

In Fig. 4(a) we plot $M$ versus $d$ for the system in Fig. 1 with fixed $d_{a}=0.9$ at varied $l_{r}=0.00025,0.1,0.025$, $0.25,40,175$, and 1750 . The overall mobility decreases with increasing $l_{r}$, while commensuration effects only appear once $l_{r}>10$, which coincides with the running length at which self-clustering begins to occur. The commensuration effects become sharper as $l_{r}$ increases. For large $d$ and large $l_{r}$, the mobility drops to zero when the system enters an active jammed or clogged state, while for smaller $l_{r}$ at high $d$, the flow is reduced but remains finite.

Figure 4(b) shows $M$ versus $d$ for the system in Fig. 1 with fixed $l_{r}=175$ and varied active disk diameter of $d_{a}=0.7,0.8,0.9$, and 1.0, giving $\phi_{a}=0.195,0.254$, 0.32 , and 0.4 , respectively. For $\phi_{a}=0.195$, no clustering occurs until $d>1.5$, which correlates with a drop in $M$ when a commensurate state forms with a structure that is similar to that illustrated in Fig. 33(c). The onset of this 
locally square commensurate state shifts to lower values of $d$ with increasing $d_{a}$. The drop in $M$ near $d=1.0$ in the $\phi_{a}=0.254$ system is due to the appearance of a different type of commensurate clustering state where the ordering is triangular rather than square, similar to what is shown in Fig. 2(b) and Fig. [3(a). The triangular commensurate state persists up to $d=1.1$ for the $\phi_{a}=$ 0.254 system and appears over a slightly higher range of $d$ in the $\phi_{a}=0.32$ system. The peak near $d=1.1$ for $\phi_{a}=0.254$ is the result of the formation of a frustrated state of the type illustrated in Fig.2(c) and Fig. 33(b). For $\phi_{a}=0.4$, the triangular commensurate state is present near the $\operatorname{dip}$ in $M$ at $d=0.7$. In general, as $\phi_{a}$ increases, the overall magnitude of $M$ drops.

The behavior of $M$ versus $d$ for samples with $l_{r}=175$ where we hold the active disk diameter fixed at $d_{a}=0.8$ but consider different disk densities $\phi_{a}=0.00212,0.09$, $0.17,0.254$, and 0.332 is shown in Fig. 4(c). Since the disk radius is fixed, the locally square commensuration $\operatorname{dip}$ in $M$ at $d=1.85$ does not shift with changing $\phi_{a}$. For $\phi_{a}=0.00212$, the system is in the single particle limit, there are no commensurate peaks or dips, and $M$ drops to zero for $d>2.25$ when the obstacles form a percolating barrier to motion. For $\phi_{a}=0.09$ and 0.17 , the triangular commensuration $\operatorname{dip}$ at $d=1.0$ and the incommensuration peak at $d=1.1$ found for larger $\phi_{a}$ are absent; however, there is a high density incommensuration peak at $d=1.95$. When $\phi_{a}=0.332$, the overall value of $M$ decreases and additional incommensuration peak forms at $d=1.5$. Here, $M$ drops to zero for $d>1.9$ when the system enters an active clogged state.

In Fig. 4(d) we plot the largest cluster size $C$ versus $d$ for the system in Fig. 4)(c) with varied $\phi_{a}$. When $\phi_{a}=0.00212, C$ remains small indicating the lack of any clustering in the single particle limit, while for $\phi_{a}=0.09$ and $\phi_{a}=0.18, C$ approaches $C=1.0$ for $d>2.25$ and has a peak at $d=1.8$ corresponding to the formation of local square ordering in individual substrate plaquettes. At $\phi_{a}=0.254$, there are three peaks in $C$ corresponding to the commensuration effects at $d=1.0,1.35$, and 1.85 , as well as a dip produced by a frustrated state at $d=1.1$. For $\phi_{a}=0.332$, a clustered state appears for all values of $d$ which develops crystalline ordering at the three commensuration values of $d$.

Summary - We have examined run-and-tumble active matter disks interacting with a periodic obstacle array and find novel active matter commensuration and frustration effects. These arise when the active matter undergoes motility-induced phase separation into a dense crystalline phase which has a natural disk spacing. When this spacing is commensurate with the lattice constant of the obstacle array, a large crystalline phase separated state can appear, whereas for other obstacle spacings, the crystalline phase cannot fit on the substrate and we instead find a frustrated state in which the clusters are amorphous and not as large. The commensuration and incommensuration effects produce peaks and dips in the mobility, six-fold order, and cluster size as a function of changing obstacle diameter. The commensurate crystal states can have long range triangular ordering or local square ordering. At low activity or in the Brownian limit, the commensuration effects are lost.

We gratefully acknowledge the support of the U.S. Department of Energy through the LANL/LDRD program for this work. This work was supported by the US Department of Energy through the Los Alamos National Laboratory. Los Alamos National Laboratory is operated by Triad National Security, LLC, for the National Nuclear Security Administration of the U. S. Department of Energy (Contract No. 892333218NCA000001).

[1] P. Bak, "Commensurate phases, incommensurate phases and the devil's staricase," Rep. Prog. Phys. 45, 587-629 (1982).

[2] S. N. Coppersmith, D. S. Fisher, B. I. Halperin, P. A. Lee, and W. F. Brinkman, "Dislocations and the commensurate-incommensurate transition in two dimensions," Phys. Rev. B 25, 349-363 (1982)

[3] C. R. Woods, L. Britnell, A. Eckmann, R. S. Ma, J. C. Lu, H. M. Guo, X. Lin, G. L. Yu, Y. Cao, R. V. Gorbachev, A. V. Kretinin, J. Park, L. A. Ponomarenko, M. I. Kastnelson, Yu. N. Gornostryev, K. Watanabe, T. Taniguchi, C. Casiraghi, H.-J. Gao, A. K. Geim, and K. S. Novoselov, "Commensurate-incommensurate transition in graphene on hexagonal boron nitride," Nature Phys. 10, 451 (2014)

[4] K. Harada, O. Kamimura, H. Kasai, T. Matsuda, A. Tonomura, and V. V. Moshchalkov, "Direct observation of vortex dynamics in superconducting films with regular arrays of defects," Science 274, 1167-1170 (1996)

[5] C. Reichhardt, C. J. Olson, and F. Nori, "Commensurate and incommensurate vortex states in superconductors with periodic pinning arrays," Phys. Rev. B 57, 7937-7943 (1998)

[6] G. R. Berdiyorov, M. V. Milošević, and F. M. Peeters, "Novel commensurability effects in superconducting films with antidot arrays," Phys. Rev. Lett. 96, 207001 (2006).

[7] S. Tung, V. Schweikhard, and E. A. Cornell, "Observation of vortex pinning in Bose-Einstein condensates," Phys. Rev. Lett. 97, 240402 (2006).

[8] M. Brunner and C. Bechinger, "Phase behavior of colloidal molecular crystals on triangular light lattices," Phys. Rev. Lett. 88, 248302 (2002).

[9] C. Reichhardt and C. J. Olson Reichhardt, "Ordering and melting in colloidal molecular crystal mixtures," Phys. Rev. E 71, 062403 (2005).

[10] T. Brazda, A. Silva, N. Manini, A. Vanossi, R. Guerra, E. Tosatti, and C. Bechinger, "Experimental observation of the Aubry transition in two-dimensional colloidal monolayers," Phys. Rev. X 8, 011050 (2018).

[11] A. Ortiz-Ambriz and P. Tierno, "Engineering of frustration in colloidal artificial ices realized on microfeatured 
grooved lattices," Nature Commun. 7, 10575 (2016)

[12] I. Bloch, "Ultracold quantum gases in optical lattices," Nature Phys. 1, 23 (2005).

[13] T. Bohlein, J. Mikhael, and C. Bechinger, "Observation of kinks and antikinks in colloidal monolayers driven across ordered surfaces," Nature Mater. 11, 126-130 (2012).

[14] A. Vanossi, N. Manini, and E. Tosatti, "Static and dynamic friction in sliding colloidal monolayers," Proc. Natl. Acad. Sci. (USA) 109, 16429-16433 (2012)

[15] C. Reichhardt and C. J. Olson Reichhardt, "Depinning and nonequilibrium dynamic phases of particle assemblies driven over random and ordered substrates: a review," Rep. Prog. Phys. 80, 026501 (2017)

[16] D. McDermott, J. Amelang, C. J. Olson Reichhardt, and C. Reichhardt, "Dynamic regimes for driven colloidal particles on a periodic substrate at commensurate and incommensurate fillings," Phys. Rev. E 88, 062301 (2013).

[17] M. Baert, V. V. Metlushko, R. Jonckheere, V. V. Moshchalkov, and Y. Bruynseraede, "Composite flux-line lattices stabilized in superconducting films by a regular array of artificial defects," Phys. Rev. Lett. 74, 3269-3272 (1995)

[18] M. C. Marchetti, J. F. Joanny, S. Ramaswamy, T. B. Liverpool, J. Prost, M. Rao, and R. A. Simha, "Hydrodynamics of soft active matter," Rev. Mod. Phys. 85, 1143-1189 (2013)

[19] C. Bechinger, R. Di Leonardo, H. Löwen, C. Reichhardt, G. Volpe, and G. Volpe, "Active particles in complex and crowded environments," Rev. Mod. Phys. 88, 045006 (2016).

[20] J. Palacci, S. Sacanna, A. P. Steinberg, D. J. Pine, and P. M. Chaikin, "Living crystals of light-activated colloidal surfers," Science 339, 936-940 (2013)

[21] I. Buttinoni, J. Bialké, F. Kümmel, H. Löwen, C. Bechinger, and T. Speck, "Dynamical clustering and phase separation in suspensions of self-propelled colloidal particles," Phys. Rev. Lett. 110, 238301 (2013)

[22] A. Morin, N. Desreumaux, J.-B. Caussin, and D. Bartolo, "Distortion and destruction of colloidal flocks in disordered environments," Nature Phys. 13, 63-67 (2017)

[23] Y. Fily and M. C. Marchetti, "Athermal phase separation of self-propelled particles with no alignment," Phys. Rev. Lett. 108, 235702 (2012).

[24] G. S. Redner, M. F. Hagan, and A. Baskaran, "Struc- ture and dynamics of a phase-separating active colloidal fluid," Phys. Rev. Lett. 110, 055701 (2013).

[25] M. E. Cates and J. Tailleur, "Motility-induced phase separation," Annual Review of Condensed Matter Physics 6, 219-244 (2015).

[26] C. Reichhardt and C. J. Olson Reichhardt, "Active microrheology in active matter systems: Mobility, intermittency, and avalanches," Phys. Rev. E 91, 032313 (2015)

[27] C. Reichhardt and C. J. Olson Reichhardt, "Active matter transport and jamming on disordered landscapes," Phys. Rev. E 90, 012701 (2014).

[28] A. Morin, D. Lopes Cardozo, V. Chikkadi, and D. Bartolo, "Diffusion, subdiffusion, and localization of active colloids in random post lattices," Phys. Rev. E 96, 042611 (2017).

[29] M. Zeitz, K. Wolff, and H. Stark, "Active Brownian particles moving in a random Lorentz gas," Eur. Phys. J. E 40, 23 (2017).

[30] T. Bhattacharjee and S. S. Datta, "Confinement and activity regulate bacterial motion in porous media," Soft Matter 15, 9920 (2019)

[31] O. Chepizhko and T. Franosch, "Ideal circle microswimmers in crowded media," Soft Matter 15, 452-461 (2019)

[32] A. Chardac, S. Shankar, M. C. Marchetti, and D. Bartolo, "Emergence of dynamic vortex glasses in disordered polar active fluids," arXiv e-prints , arXiv:2002.12893.

[33] G. Volpe, I. Buttinoni, D. Vogt, H.-J. Kümmerer, and C. Bechinger, "Microswimmers in patterned environments," Soft Matter 7, 8810-8815 (2011).

[34] R. Alonso-Matilla, B. Chakrabarti, and D. Saintillan, "Transport and dispersion of active particles in periodic porous media," Phys. Rev. Fluids 4, 043101 (2019).

[35] H. E. Ribeiro, W. P. Ferreira, and Fabrício Q. Potiguar, "Trapping and sorting of active matter in a periodic background potential," Phys. Rev. E 101, 032126 (2020)

[36] M. Brun-Cosme-Bruny, A. Förtsch, W. Zimmermann, E. Bertin, P. Peyla, and S. Rafaï, "Deflection of phototactic microswimmers through obstacle arrays," Phys. Rev. Fluids 5, 093302 (2020).

[37] S. Yazdi, J. L. Aragones, J. Coulter, and A. AlexanderKatz, "Metamaterials for active colloid transport," arXiv e-prints , arXiv:2002.06477.

[38] C. Reichhardt and C. J. O. Reichhardt, "Directional locking effects for active matter particles coupled to a periodic substrate," Phys. Rev. E , in press (2020). 